\title{
Complémentations alimentaires en élevage semi-extensif sur savanes soudano-guinéennes d'altitude au Cameroun
}

\author{
par J. PIOT (*)
}

\begin{abstract}
RESUME
L'auteur, dans cet article, relate des essais de complémentation simples applicables à des bovins en troupeau.

Les 2 premiers essais concernent une complémentation de saison sèche qui vise à maintenir les animaux au moins dans leur meilleur état de saison des pluies.

Le troisième essai concerne une complémentation de saison des pluies propre à mieux valoriser la production herbagère dans certaines conditions.

Ces complémentations, qui peuvent se faire avec du tourteau de coton ou de la graine de coton, sont susceptibles de vulgarisation en élevage traditionnel, ce qui n'est pas le cas des schémas d'embouche intensive rapide.
\end{abstract}

Les conjonctures économiques et sociales de l'Afrique deviennent de plus en plus favorables à des possibilités de production intensive de viande bovine. Les multiples publications d'essais d'embouche intensive déjà parues sont la preuve la plus évidente de cette évolution.

Pourtant, dans l'état actuel des choses, il s'agit essentiellement de préciser les techniques d'embouches et les aptitudes des différentes races africaines disponibles, à valoriser les rations offertes.

Il reste néanmoins deux obstacles majeurs:

1. La difficulté de rentabiliser une embouche courte, car même en espérant une augmentation sensible du prix de la viande, les prix des aliments de bases (Sorgho et maïs en Adamaoua) se sont déjà accrus considérablement.

2. Dans une région d'élevage traditionnellement extensif, l'éleveur, même s'il devient

(*) C.R.Z., Station fourragère, B.P. 50, Wakwa, Cameroun. engraisseur, restera avant tout à la tête du circuit sous forme de naisseur ou fournisseur de " maigre ".

Cependant, si les troupeaux sont très mouvants, une bonne partie des propriétaires sont sédentaires et capables de garder auprès d'eux des noyaux de bétail particuliers (vaches à traire près des centres urbains par exemple). Mais la complexité des méthodes d'embouche intensive, l'importance des investissements de base et du contrôle mettent tout cela hors de portée de l'éleveur traditionnel.

Depuis plusieurs années, la Station Fourragère I.E.M.V.T. de Wakwa (NGaoundéré) effectue des essais de complémentation aussi simples que possible, réalisables sans infrastructure lourde sur des animaux restant par ailleurs constitués en troupeaux normaux. Cela pour souligner l'élimination des énormes sujétions matérielles que représentent les distributions individuelles de rations de concentrés en stalles par exemple. 
En général, les complémentations ont été effectuées en saison défavorable plus ou moins par la nécessité d'aider les animaux déficients. Un essai d'embouche de type long avec complémentation de saison des pluies sera cependant également relaté.

Dans tous les cas, les essais décrits ici se sont faits sur les pâturages naturels à Hyparrhenia Spp. des savanes arborées et arbustives à Daniellia oliveri Hutch. et Dalz. et Lophira lanceolata Van Tieg. classiques en Adamaoua (5).

Les compléments utilisés ont été ceux que l'on peut le plus facilement se procurer localement à savoir, tourteaux de coton et graines de coton. Ce dernier produit manque d'intérêt en raison des aléas des approvisionnements : récoltes trop irrégulières, quantités triturées en huilerie de plus en plus importantes, livraison en fin de saison sèche qui oblige à des stockages longs et difficiles jusqu'à la saison sèche suivante. au plus mauvais moment. Il faut alors à ces animaux (vaches et veaux) des régimes privilégiés pour leur conserver des chances de survie.

Ce même type de complémentation de saison sèche est également intéressant pour essayer de conserver leur bon état à des animaux destinés à être abattus pendant cette période. C'est alors de 15 à 20 p. $100 \mathrm{du}$ poids que l'on préservera comme l'ont montré les études effectuées à la Station Fourragère sur les mêmes pâturages que ceux où se sont effectués les essais relatés ensuite.

LHOSTE (3) confirme ces nombres en annonçant 11 p. 100 entre le 27 janvier et le 21 avril sur une savane plus riche et les 2 premiers mois de saison sèche non pris en considération.

Mais précisons tout d'abord la valeur alimentaire de ce qui peut être offert au bétail.

Les pâturages naturels sont en saison sèche d'une pauvreté assez marquée et le tableau ci-dessous le montre bien $(3,6)$.

TABL. $N^{\circ} I-V a l e u r$ de $I^{\prime}$ herbe sur pied disponible en saison sèche

\begin{tabular}{|l|c|c|c|c|c|}
\hline & M.S. & $\begin{array}{r}\text { Valeur énergétique } \\
\text { UF/kg de M.S. }\end{array}$ & $\begin{array}{c}\text { M.P.D. } \\
\text { g/kg de M.S. }\end{array}$ & $\frac{\text { M.P.D. }}{\text { U.F. }}$ & $\frac{\text { M.S. }}{\text { U.F. }}$ \\
\hline $\begin{array}{l}\text { Fourrage sur pied } \\
\text { Savanes naturelles }\end{array}$ & 67 & 0,4 & 10 & 25 & 2,5 \\
\hline $\begin{array}{l}\text { Fourrage sur pied } \\
\text { Savane amêliorée }\end{array}$ & 63 & 0,45 & 12 & 27 & 2,2 \\
\hline $\begin{array}{l}\text { Brachiaria brizantha } \\
\text { sur pied }\end{array}$ & 68,35 & 0,39 & 5 & 13 & 2,6 \\
\hline $\begin{array}{l}\text { Repousses de saison } \\
\text { sêche sur Savanes } \\
\text { naturelles sans feux }\end{array}$ & 16,45 & 0,52 & 18 & 35 & 1,9 \\
\hline
\end{tabular}

M.S. = Matière sèche; M.P.D. = Matières Protéiques Digestibles

\section{COMPLEMENTATIONS DE SAISON SECHE}

C'est ce type de complémentation qui s'impose le premier à l'éleveur soucieux du maintien en état de ses animaux qui sont en premier lieu les vaches suitées plus ou moins épuisées.

Il n'est pas rare de retrouver en fin de saison sèche ce type d'animaux à plus de 30 p. 100 en dessous de son poids avant vêlage. C'est systématiquement le cas pour une vache qui mettra bas à l'époque la plus défavorable (septembre, octobre, novembre), ce qui la mettra avec un veau exigeant, pas encore sevré,
Surtout, apparaît le très important déficit protéinique à peine amélioré par un brout arbustif, d'ailleurs pas toujours possible, et une maigre repousse de saison sèche vite épuisée.

Les études plus approfondies $(4,6)$ montrent que, du point de vue minéral, la faible teneur en phosphore abaisse le rapport $\mathrm{P} / \mathrm{Ca}$ à un niveau trop faible cependant que l'excès de potassium mérite d'être corrigé par un apport de CINa.

Les compléments alimentaires disponibles ont leurs valeurs résumées dans le tableau II. 
TABLEAU $\mathrm{N}^{\circ} \mathrm{II}$-Valeur alımentaire des compléments utilisếs.

\begin{tabular}{|c|c|c|c|c|c|c|c|c|c|c|}
\hline $\begin{array}{c}\text { Valeurs pour } \\
1 \mathrm{~kg} \mathrm{~d} a l \text { iment }\end{array}$ & M.S. & M.P.B. & Cellulose & $\begin{array}{l}\text { Matières } \\
\text { grasses }\end{array}$ & $\mathrm{Ca}$ & $P$ & K & $\mathrm{Mg}$ & UF & M.P.D. \\
\hline Tourteau arachide & 934,5 & 524 & 47,5 & 66,9 & 2,06 & 7,24 & 13,7 & 3,6 & 1 & 400 \\
\hline Tourteau coton & 930,5 & 525 & 36,5 & 55,3 & 1,3 & 6,52 & 8,5 & 3,4 & 1 & 350 \\
\hline Graines de coton & 935 & 205 & 264 & 199 & 1,4 & 6,36 & 10,5 & 3,37 & 1,03 & 125 \\
\hline $\begin{array}{l}\text { Graines de coton } \\
\text { diteo "jaune" }\end{array}$ & 929,8 & 176,3 & 300 & 126,4 & 1,35 & 4,94 & 12,6 & 3,31 & 1 & 106 \\
\hline
\end{tabular}

M.P.B. = Matières Protéiques Brutes.

Ce sont :

\section{- Tourteau de coton}

Nous disposons d'un très bon produit riche en matière azotée dont l'indice de Léa demeure très correct $(18,5)$ même après un stockage de quelques mois. Cette qualité de tourteau est variable avec les approvisionnements en restant toutefois à un niveau de qualité élevé.

\section{- Tourteau d'arachide}

Ce produit, également de haute valeur, est devenu rare au Cameroun et nous devons le remplacer systématiquement par le tourteau de coton.

\section{- Graine de coton}

En année de production normale, cette graine non délintée est excellente. Nous n'avons pu disposer, après la mauvaise récolte 1970, que de graines " jaunes " non triturables, moins riches en matières protéiniques.

\section{- Perlurée engrais à 46 p. 100 d'azote}

L'urée alimentaire réputée plus efficace est localement difficile à obtenir et nous nous contentons de cette présentation.

Enfin, il nous reste, en guise de généralités, à préciser les besoins alimentaires de nos animaux et nous adoptons pour cela les normes admises pour l'animal de référence indiquées par BOUDET et RIVIERE (1).

L'animal de référence est le Zébu de $250 \mathrm{~kg}$ (U.B.T.) qui peut consommer par jour $6,250 \mathrm{~kg}$ de matière sèche et qui, pour de petits déplacements, exige une ration d'entretien de 2,7 unités fourragères et de $151 \mathrm{~g}$ de matières protéiques digestibles.

Les croîts se font à raison de 0,33 U.F. et $17,5 \mathrm{~g}$ de M.P.D. pour $100 \mathrm{~g}$ de gain vif.

\section{Essai préliminaire}

Les circonstances nous ont conduit, en saison sèche 1967-68, à complémenter un bœuf isolé du troupeau où il se trouvait à la saison des pluies 1967.

Le diagramme $n^{*} 1$ montre ce qu'a été le comportement de cet animal recevant journellement :

- $250 \mathrm{~g}$ de tourteau de coton;

- $500 \mathrm{~g}$ de tourteau d'arachide;

- 25 g de perlurée.

La courbe inférieure correspond au poids moyen par tête des dix autres animaux de ce troupeau conservés comme témoins sur pâturage riche en refus. La charge sur un hectare était ramenée à $125 \mathrm{~kg} / \mathrm{ha}$.

Sur la base des besoins de l'animal de référence (1) les calculs nous ont persuadé que nous devions assurer en totalité les besoins protéiniques.

Dans ces conditions, un rapide bilan a révélé l'aspect positif de cette complémentation :

A $45000 \mathrm{~F}$ la tonne de perlurée et $19 \mathrm{~F}$ le $\mathrm{kg}$ de tourteau, l'intervention coûte 16 F CFA par jour en aliment, soit $1900 \mathrm{~F}$ pour la saison; ceci est à opposer à la conservation de poids vif de $70 \mathrm{~kg}$, ce qui à $60 \mathrm{~F} \mathrm{CFA}$ environ le $\mathrm{kg}$ représente donc plus de $4000 \mathrm{~F} \mathrm{CFA.}$

Ce premier essai nous a convaincu de ce que l'on pouvait au moins empêcher les animaux de maigrir en saison sèche sur pâturage non brâlé pourvu que leurs besoins protéiniques soient satisfaits. Conserver pendant la saison défavorable le bétail dans le meilleur état atteint en saison des pluies constitue déjà une méthode de production de viande.

Un essai plus large devait confirmer l'approche citée précédemment. 

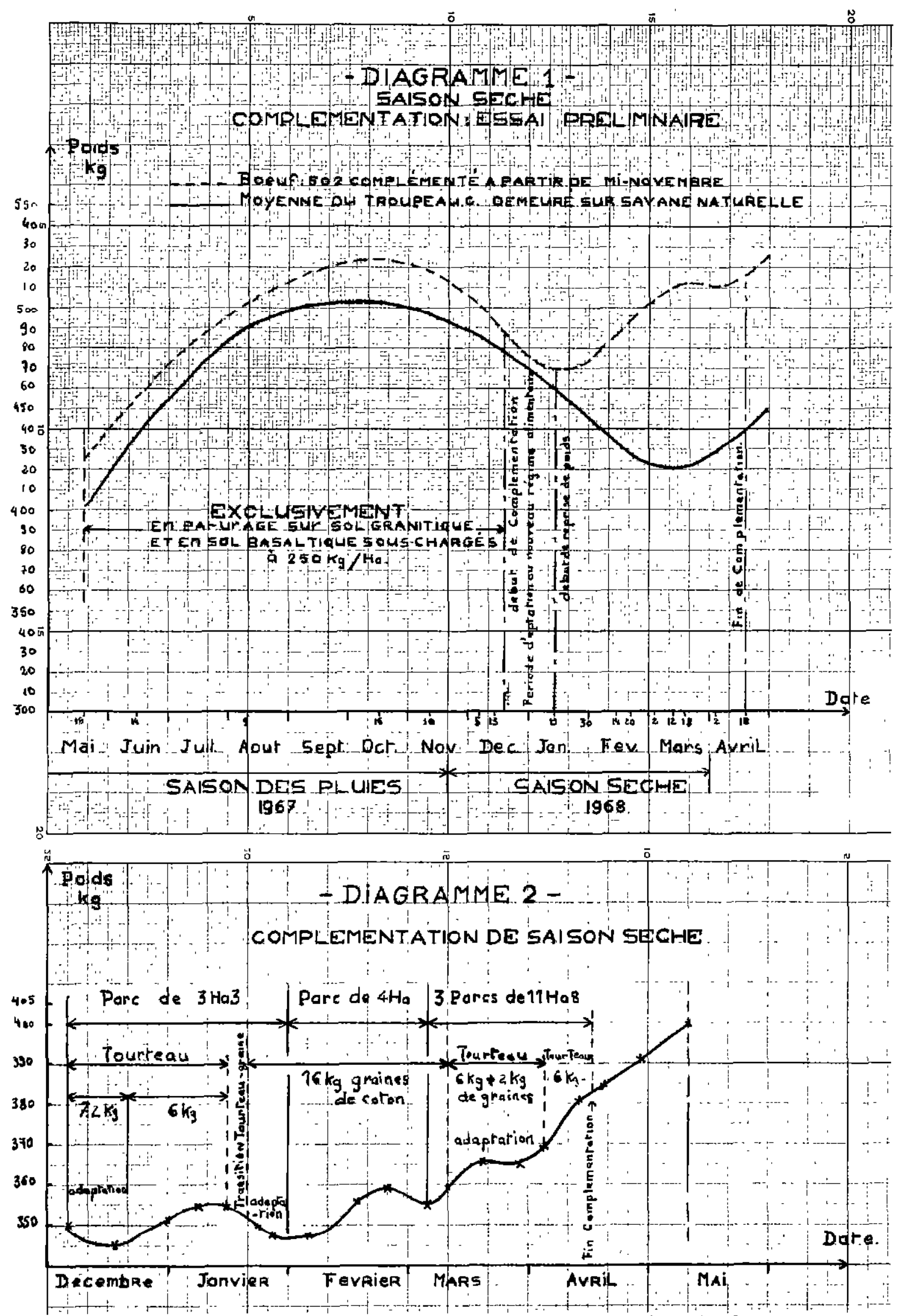

Ce diagramme montre l'évolution pondérale moyenne d'un troupeau de 10 boeufs recevant au total la complémentation indiquée. On remarque qu'à chaque modification de la ration correspond une perte de poids même lorsque le changement est très progressif. 
Six bœufs de race Foulbé sont comparés à 4 boufs de race Wakwa (1/2 sang FoulbéBrahman de $2^{e}$ génération ou plus).

Le diagramme 2 précise les conditions et le déroulement de l'expérience ainsi que le tableau III qui peut être utile au technicien qui aura à réaliser une telle complémentation.

Les changements de rations sont liés aux irrégularités d'approvisionnement auxquelles nous nous sommes heurté car nous souhaitions faire l'expérience uniquement avec de la graine de coton. Compte tenu des quantités plus considérables qu'il convient d'offrir aux animaux que lorsqu'il s'agit de tourteau, il y a moins de risque de voir certains d'entre eux complètement écartés des aliments distribués. En distribution collective, ce point est important pour obtenir un comportement individuel assez homogène.

Le pâturage est constitué par la masse des refus de parcs basaltiques qui n'ont été chargés qu'à $250 \mathrm{~kg} / \mathrm{ha}$ la saison des pluies précédente. (Il faut des charges de l'ordre de $500 \mathrm{~kg} / \mathrm{ha}$ de mai à décembre pour avoir des parcs sans refus.)

Les animaux pèsent $350 \mathrm{~kg}$ en moyenne au début et leurs besoins en M.P.D. ne sont que de $210 \mathrm{~g}$ /jour pour l'entretien puisque les déplacements sont très limités (1).

Nous avons donc estimé qu'il fallait apporter $600 \mathrm{~g}$ de tourteau de coton ou $1,6 \mathrm{~kg}$ de graines de coton par animal et par jour.

Un complément minéral était mélangé aux aliments comportant $15 \mathrm{~g}$ de $\mathrm{CINa}, 15 \mathrm{~g}$ de calcyle et $2 \mathrm{~g}$ de complexe oligo-élément par animal et par jour.

- Les résultats techniques apparaissent au tableau III et sur le diagramme 2 .

On constate qu'un croît de 11,5 p. 100 a été obtenu en fin de complémentation pour les Wakwa et 8,5 p. 100 pour les Foulbé.

En début de l'expérience, les animaux ont commencé à perdre environ $9 \mathrm{~kg}$ par rapport à leur poids maximal atteint le 15 novembre.

La reprise à l'herbe après complémentation est tout de même très nette avec environ $6 \mathrm{p}$. 100 en quatre semaines, ce qui tend à confirmer qu'un avantage pris en saison défavorable se conserve en grande partie toute la vie de l'animal.
Les résultats économiques font apparaître un bilan positif intéressant.

L'apport quotidien graine ou tourteau nous coûte environ $11,50 \mathrm{~F}$ plus $1,50 \mathrm{~F}$ de compléments minéraux, soit $1600 \mathrm{~F}$ en cent vingt jours.

Le gain moyen de poids vif a été de $30 \mathrm{~kg}$, soit $16 \mathrm{~kg}$ de viande, à une époque où nous avons pu la vendre $135 \mathrm{~F}$. A ce stade, nous avons plus de $550 \mathrm{~F}$ de bénéfice par tête.

Les charges considérables des pâturages (tableau III) ne nous auraient pas permis de conserver sans mortalité un lot témoin identique en nombre à comparer au lot complémenté. Mais même à seulement $250 \mathrm{~kg} / \mathrm{ha}$, nous aurions perdu un minimum de 15 p. 100 du poids des animaux. Et c'est bien en définitive dans cette perte évitée que se trouve l'essentiel du bénéfice de l'opération.

$\mathrm{Au}$ total, c'est $3000 \mathrm{~F}$ par tête qui sont gagnés et c'est un minimum car nous négligeons la perte due au déclassement pour carcasse maigre.

Enfin, au bilan économique de telles opérations, on doit ajouter le temps gagné pour obtenir la carcasse commercialisable légalement fixée à $200 \mathrm{~kg}$. Schématiquement, c'est une année qui est gagnée avec comme corollaire :

- Une plus grande disponibilité de pâturage,

- Un risque pathologique diminué, ce qui est loin d'être négligeable comme le montre l'expérience, même dans les conditions suivies de Station où nous opérons.

La complémentation des " animaux fatigués " définis précédemment s'effectue sur les mêmes bases techniques, ce qui nous conduit à un apport de $1 \mathrm{~kg}$ de tourteau par vache suitée d'environ $350 \mathrm{~kg}$.

La justification économique essentielle dans ce cas réside dans le fait que l'on préserve le capital lui-même (vache et veau souvent) qui sinon serait condamné en partie.

Sans traiter de ces essais en détail, nous soulignerons que cette méthode, telle qu'elle, est parfaitement utilisable pourvu que l'intervention se fasse assez tôt. Une assez longue période d'adaptation des animaux à un changement de régime alimentaire est, nous l'avons vu, nécessaire. Ce laps de temps pourrait être fatal à des animaux épuisés mis en complémentation trop tardivement. 


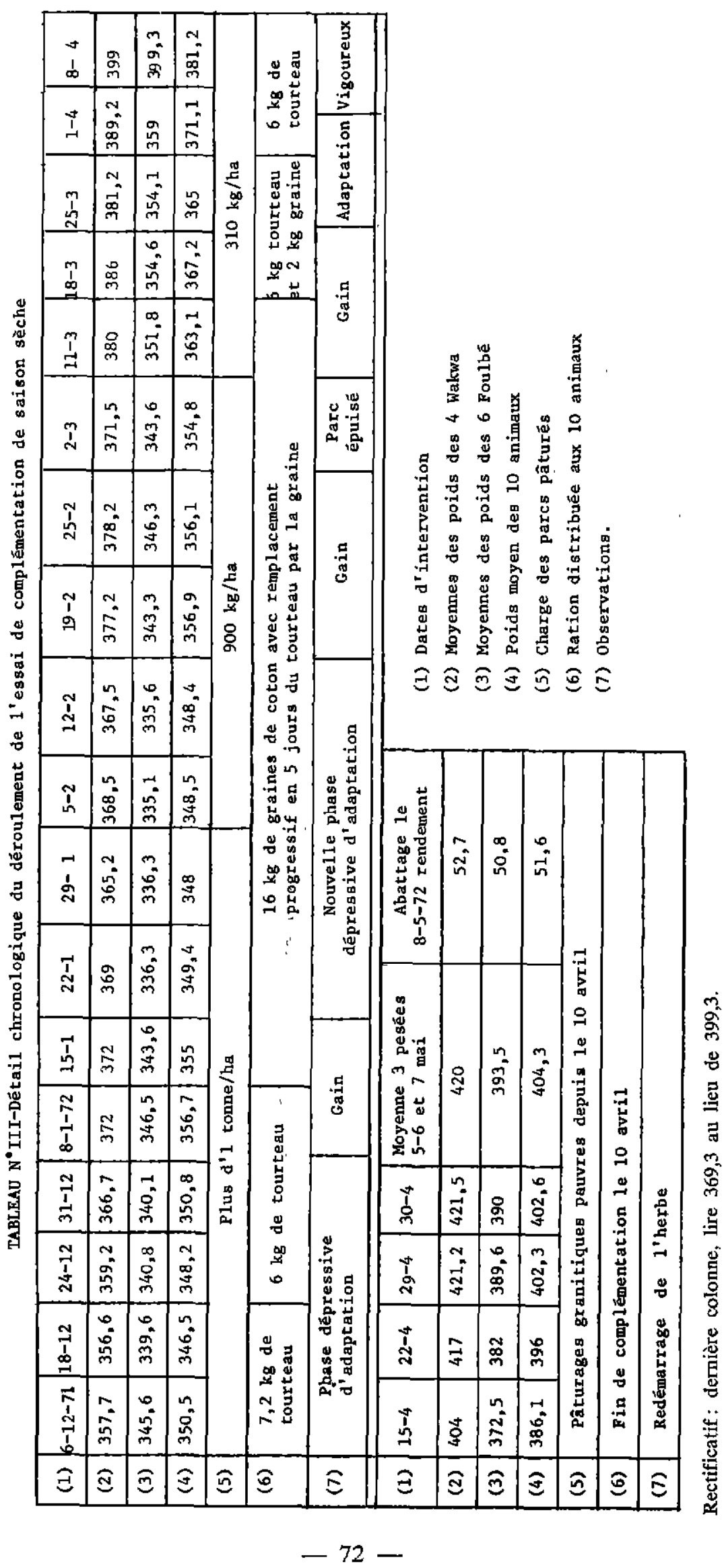




\section{COMPLEMENTATION DE SAISON DES PLUIES}

Ces essais ont des justifications assez différentes des précédents, mais visent aussi à réduire le temps d'occupation des pâturages en assurant une croissance plus rapide du bétail.

C'est essentiellement le comportement des animaux aux changements de conditions de pâture qui nous a poussé à effectuer cet essai.

Chaque année, des animaux nous sont confiés pour une saison des pluies pendant laquelle nous faisons exploiter en rotation des parcs expérimentaux.

Chaque fois, nous constatons des croissances insuffisantes surtout par rapport à des lots comparables déjà en place et conservés sur leurs terrains habituels sans changement de parc.

Mais ce fait est plus général et les grandes compagnies d'élevage le savent bien qui constatent que les animaux d'achat destinés à une embouche longue ne "font rien " la première année pendant laquelle ils se contentent de "prendre le pâturage ". Et pourtant ces animaux sont dans des conditions très proches de celles des élevages traditionnels où ils vivaient avant leur achat.

Pour des Stations de production, les seules incidences résultent d'un retard de commercialisation d'un an. Mais en Station de sélection, ce retard était inadmissible, car nos taurillons, en raison du stress provoqué par le changement de milieu, étaient déclassés à coup sûr au moment des comparaisons de sélection avec leurs homologues restés dans leur milieu habituel.

Mais, la complémentation à effectuer ne pouvant se faire au hasard, nous en avons cherché les bases dans une estimation qualitative de la ration prélevée au pâturage par les animaux au cours de la saison des pluies.

Cette expérimentation a été réalisée de la façon suivante :

4 parcs basaltiques chargés à $500 \mathrm{~kg} / \mathrm{ha}$ ont été parsemés de placeaux de $7 \mathrm{~m} \times 7 \mathrm{~m}$ protégés de la pâture par groupes de 2 pendant la période de passage du troupeau. A chaque rotation, les 2 placeaux protégés étaient différents.

Le jour de la sortie du troupeau, par prélèvement à la main en général, on recréait dans les placeaux la physionomie du pâturage environnant tel que le laissaient les animaux. Un échantillonnage de ces récoltes a fait l'objet d'analyses chimiques classiques.

Les 8 analyses qui correspondent à chaque rotation ont été regroupées pour en tirer des moyennes. Les valeurs bromatologiques des analyses chimiques ont été estimées à partir des tables hollandaises et sont récapitulées dans le tableau IV.

Dans ce tableau, nous avons présenté tout d'abord les résultats des prélèvements; ensuite, ce que devrait valoir l'herbe disponible pour des animaux pesant environ $250 \mathrm{~kg}$ de poids vif, avec un croît quotidien de $300 \mathrm{~g}$.

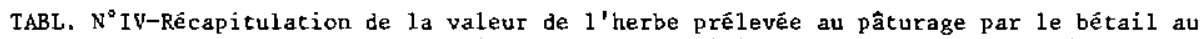
cours de la saison des pluies dans des conditions de charge de $500 \mathrm{~kg} / \mathrm{ha}$.

\begin{tabular}{|c|c|c|c|c|c|c|c|c|c|c|}
\hline \multicolumn{2}{|c|}{ Pér i ades } & $\begin{array}{c}9-5 \text { au } \\
3-6\end{array}$ & $\begin{array}{c}3-6 \text { au } \\
2 B-6\end{array}$ & $\begin{array}{c}28-6 \\
\text { au } \quad 23-7\end{array}$ & $\begin{array}{c}23-7 \\
\text { au } 17-8\end{array}$ & $\begin{array}{c}17-8 \\
\text { au } 11-9\end{array}$ & $\begin{array}{c}11-9 \\
\text { au } 6-10\end{array}$ & $\begin{array}{c}6-10 \text { au } \\
31-10\end{array}$ & $\left\{\begin{array}{c}31-10 \text { au } \\
24-11\end{array}\right.$ & $\begin{array}{c}24-11 \text { au } \\
19-12\end{array}$ \\
\hline \multirow{5}{*}{ 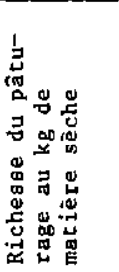 } & U.F. & 0,59 & 0,49 & 0,52 & 0,50 & 0,51 & 0,50 & 0,59 & 0,54 & 0,52 \\
\hline & MPD en $\mathrm{g}$ & 37,8 & 40 & 40 & 43 & 40 & 44 & 35 & 24 & 23 \\
\hline & $\mathrm{Ca}$ en $\mathrm{g}$ & 2,88 & 2,70 & 2,56 & 2,58 & 2,82 & 2,81 & 3.89 & 3,94 & 3,85 \\
\hline & $P$ en $B$ & 1,95 & 1,89 & 1,97 & 2,21 & 2,21 & 2,04 & 1,62 & 1,30 & 1,13 \\
\hline & MPD/UF & 64 & 82 & 77 & 86 & 79 & 88 & 60 & 45 & 44 \\
\hline \multirow{4}{*}{ 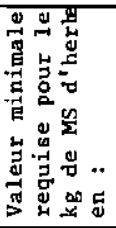 } & U.F. & 0,592 & 0.59 & 0,588 & 0,573 & 0,572 & 0,57 & 0,568 & 0,568 & 0,555 \\
\hline & MPD en $B$ & 32,6 & 32,3 & 32,1 & 31,8 & 31,7 & 31,6 & 31,3 & 31,2 & 31,1 \\
\hline & Ca en $g$ & \multicolumn{9}{|c|}{2} \\
\hline & $P$ en $B$ & \multicolumn{9}{|c|}{1,2} \\
\hline
\end{tabular}


D'une manière générale, on voit que la valeur énergétique de l'herbe est trop faible pour assurer un croît convenable.

En outre, la nette remontée du mois d'octobre liée à l'exploitation des fructifications de la plupart des graminées est parfaitement soulignée.

Dès le mois de novembre, c'est la valeur protéinique qui devient tout à fait insuffisante et demande à être corrigée.

Enfin, du point de vue des minéraux de base, les besoins sont toujours à peu près satisfaits et le seul éventuel problème qui se pose est un problème d'équilibre du rapport phosphore-calcium.

En pâturages de sols granitiques ou sur formation végétale pauvre, les baisses protéiniques et énergétiques sont marquées plus tôt et plus fortement.

Quant aux besoins alimentaires des animaux, ils sont les mêmes qu'aux essais précédemment décrits.

L'essai de complémentation a été effectué avec 52 taurillons Wakwa, Foulbé et $1 / 4$ de sang Brahman qui ont commencé les rotations le 15 mai avec une charge de départ de $400 \mathrm{~kg} / \mathrm{ha}$ sur les terrains granitiques et $500 \mathrm{~kg} / \mathrm{ha}$ sur les terrains basaltiques.

La complémentation reçue quotidiennement a été de :
- $26 \mathrm{~kg}$ de graines de coton;

- $500 \mathrm{~g}$ de $\mathrm{CINa}$;

- $500 \mathrm{~g}$ de calcyle;

- $100 \mathrm{~g}$ de complexe oligo-élément.

Cela jusqu'au 15 août. Du 15 août au 15 décembre, la quantité de graines de coton a été doublée.

Le tableau $\mathrm{V}$ donne l'évolution de notre troupeau complémenté, comparée à celle des animaux homologues demeurés en place qui dès le premier mois prennent un avantage considérable d'une vingtaine de $\mathrm{kg}$ (22 pour les Wakwa, 15,5 pour les Foulbé).

Ce handicap ne sera jamais totalement rattrapé par la complémentation mais l'expérience nous permet d'affirmer qu'il n'aurait fait que s'aggraver autrement car le croît des animaux fut, les autres années, deux fois plus faible que celui des homologues non perturbés dans leurs conditions de pâture (4). En l'occurrence, les poids au 15 novembre pour la Station Fourragère auraient été de l'ordre de $320 \mathrm{~kg}$ et $295 \mathrm{~kg}$ respectivement pour les Wakwa et les Foulbé.

En outre bien entendu, dès novembre nous empêchons la perte de poids de s'amorcer et cela complète les études précédentes de complémentation de saison sèche qui avaient débuté en décembre et n'avaient marqué que fin décembre compte tenu de la phase d'adaptation.

TABLEAU $\mathrm{N}^{\circ} \mathrm{V}$ - Evolution pondêrale

\begin{tabular}{|c|c|c|c|c|c|}
\hline \multirow[t]{3}{*}{ Troupeaux } & \multicolumn{4}{|c|}{ Poids moyens par animal } & \multirow[b]{3}{*}{ Ob ge I vat $\mathrm{i}$ o n s } \\
\hline & \multicolumn{2}{|c|}{$W a k w a$} & \multicolumn{2}{|c|}{ F o u 1 b é } & \\
\hline & $\begin{array}{c}32 \text { mâles } \\
\text { complémentës } \\
\text { (Station } \\
\text { Fourtagère) }\end{array}$ & $\begin{array}{l}10 \text { mâles } \\
\text { sélectionnés } \\
\text { (Station } \\
\text { zootechnique) }\end{array}$ & $\begin{array}{c}20 \text { mâles } \\
\text { complémentés } \\
\text { (Station } \\
\text { Fourragère) }\end{array}$ & $\begin{array}{l}17 \text { mâles } \\
\text { sélectionnés } \\
\text { (Station } \\
\text { Zootechnique) }\end{array}$ & \\
\hline mi avril & 226,5 & 232,5 & 209 & 230 & Les animaux sont ensemble \\
\hline 11 mai & 243 & 250 & 225 & 246,5 & \\
\hline 15 mai & 250 & & 231,5 & & $\begin{array}{l}\text { Arrivée des animaux à } 1 \mathrm{a} \\
\text { Station Fourragère }\end{array}$ \\
\hline $23 \mathrm{mai}$ & & 274 & & 276 & \\
\hline 20 juin & 261 & 290,5 & 242 & 280 & \\
\hline 15 novembre & 346 & 367 & 321 & 353 & \\
\hline 15 dëcembre & 346 & 357,5 & 322 & 346,5 & $\begin{array}{l}\text { Fin de complémentation à la } \\
\text { Station Fourragère }\end{array}$ \\
\hline
\end{tabular}




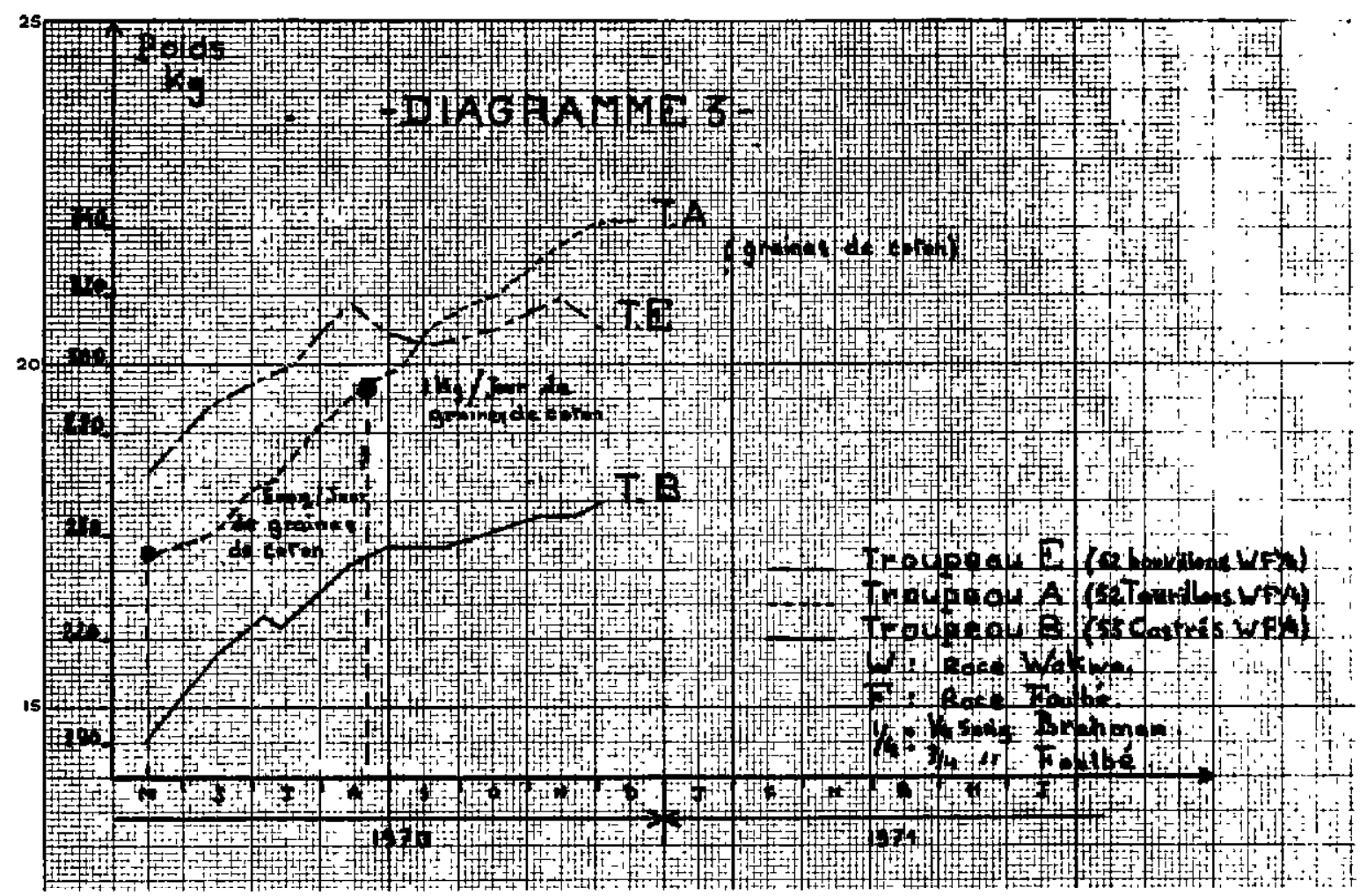

Diagramme 3 d'évolution pondérale d'animaux dans des conditions de pâture très voisines mais avec complémentation (A) ou sans.

- Le troupeau $\mathrm{E}$ accuse la baisse de valeur du pâturage du mois d'août et la remontée due aux fructifications en octobre.

- Le troupeau B décroche moins en août grâce à son potentiel de croissance supérieur lié à l'âge moins élevé des animaux.

On remarque les variations de poids très comparables jusqu'au mois d'août.

Le diagramme 3 résume l'évolution pondérale du troupeau étudié (A). Il permet la comparaison avec d'autres troupeaux ( $E$ surtout) menés dans des conditions de pâture comparables mais sans complémentation.

Du point de vue pécuniaire, la complémentation nous a coûté 8,5 tonnes de graines de coton, soit environ $60000 \mathrm{~F}$. En échange, nous avons pu maintenir le troupeau sur une trentaine d'hectares de qualité moyenne à médiocre au lieu des 60 à 65 hectares de bonne qualité qu'il aurait exploité dans sa Station d'origine pour donner les mêmes résultats.

Tout se passe donc comme si nous avions accepté " de louer " du pâturage $2000 \mathrm{~F}$ l'hectare.

Signalons que ce schéma, où la graine est remplacée par du tourteau et de la farine de céréales, a été utilisé par une grande société d'élevage pour préparer à l'abattage de saison sèche des lots de bœufs pour lesquels au moins une année a été gagnée.

\section{CONCLUSIONS}

Ces divers types de complémentation sont très simples à mettre en œuvre. Ils sont donc accessibles à bien des éleveurs avertis, simplement avec l'aide de leurs bergers.

Et si l'idée de complémentation de saison des pluies est encore prématurée, celle de saison sèche s'est déjà implantée dans les esprits d'éleveurs dynamiques en Adamaoua. Les services de vulgarisation ont en effet installé avec un succès indéniable des points de démonstration en milieu traditionnel sur les bases des essais de saison sèche relatés dans cet article.

Des lots d'animaux destinés à la boucherie et à maintenir en état ont été concernés ainsi que des lots de vaches suitées. 
Le schéma tourteau - fourrage sur pied a été retenu pour les secteurs non brûlés et en secteur brûlé, le grossier a été remplacé par du foin de Stylosanthes assez pauvre en moyenne (perte de folioles importante due aux manipulations).

Ce qui semble le plus avoir séduit les éleveurs dans ces opérations est l'absence de perte d'animaux qu'ils ont pu conserver près d'eux et surveiller. Les transhumances se traduisent toujours par des pertes élevées durement ressenties et il est normal que ce résultat très concret soit mieux perçu par l'éleveur que l'avantage pondéral moins visible.

Mais pour la vulgarisation, il y a un problème de disponibilités financières de l'éleveur, délicat à résoudre à cause surtout de la " fluidité " de son patrimoine.

Il semble alors que l'on s'orienterait vers une diminution du prix du tourteau, la différence étant probablement couverte par des subventions.

Du point de vue économique, un prix trop bas du tourteau peut présenter un risque de gaspillage de ce sous-produit finalement pas très abondant. La production camerounaise permet de complémenter 100000 à 200000 têtes de bétail par an suivant la nature du bétail concerné. Nous avons montré que tout l'intérêt de cette complémentation résidait dans l'apport protéinique qui doit être assuré en quantité juste suffisante. Il ne faudrait pas qu'un prix trop bas soit un encouragement à satisfaire aussi une part importante des besoins énergétiques. Il y aurait alors un gaspillage azoté certain et une moindre possibilité de production animale totale.
Du point de vue technique, nous insisterons encore sur l'importance et la longueur des périodes d'adaptation, alimentaire du bétail chaque fois que la composition de la ration est modifiée. Cela est très net dans notre essai de saison sèche exagérément perturbé contre notre gré. Et cependant nous avions prévu des transitions très progressives qui se sont malgré tout soldées par des pertes de poids très nettes.

Nous soulignerons aussi que les reprises de poids au retour de la saison des pluies sont très marquées même si les animaux ne sortent pas d'une période d'amaigrissement. Le poids ainsi conservé sur les animaux constitue donc bien un bénéfice acquis.

Enfin, nous noterons tout l'intérêt que représentent les normes et tables alimentaires utilisées pour l'U.B.T. Quelles que soient les imperfections reconnues de ces documents, nous avons trouvé en eux des bases de travail bien suffisantes eu égard à la "finesse " possible des réalisations dans les conditions de l'élevage extensif.

Les savanes de l'Adamaoua sont des zones pastorales riches qui méritent beaucoup mieux qu'une simple cueillette. De plus, les pâturages disponibles sont pour différentes raisons (dégradation, pathologie), pratiquement irrécupérables à bref délais par l'éleveur traditionnel. Il est donc évident que dans ces conditions, le " fond " exploitable prend une forme sensible et commence à être considéré par l'éleveur luimême comme source tangible de sa production animale. Toutes les techniques qui se présenteront propres à gagner du temps dans l'élevage du bétail et donc à libérer plus rapidement les pâturages recevront des éleveurs un accueil de plus en plus favorable.

\section{SUMMARY}

\section{Supplementary feeding in semi extensive cattle rearing} on high altitude sudan gumean savanna in Cameroon

In this paper, the author reports on trials of simple supplementary feeding on herds of cattle.

The two first trials concern supplementation in the dry season directed towards maintaining the animals at least in their best state of the wet season.

The third trial deals with supplementation during the rainy season to make the best use of grassland production under some conditions.

These supplementations, which can be such material as cotton cakes or cotton seeds, can be extended in traditional management, which is not the case for the patterns of rapid intensive fattening. 


\section{RESUMEN}

Aditivos alimenticios en ganaderia semi-extensiva sobre sabanas sudano-guíneanas de altítud en Camerún

Se hicieron ensayos simples de alimentación con aditivos para bovinos viviendo en manada.

Los dos primeros ensayos conciernen una alimentación con aditivos durante la estación seca, con el objeto de mantener los animales por lo menos en su mejor estado existiendo durante la estación de las lluvias.

En el tercero ensayo se tratan de aditivos utilizados durante la estación de las lluvias para valorizar más la producción forrajera en ciertas condiciones.

Estos aditivos, torta de algodón o grano de algodón, pueden ser vulgarizados en lo concerniente a la ganaderia tradicional, lo que no es el caso de los programas de engorde intensivo rapido.

\section{BIBLIOGRA PHIE}

1. BOUDET (G.) et RIVIERE (R.). Emploi pratique des analyses fourragères pour l'appréciation des pâturages tropicaux. Rev. Elev. Méd. vét. Pays trop., 1968, 21 (2): 227-266.

2. JOUVE (J. L.) et LETENNEUR (L.). Etude en Côte d'Ivoire, de la croissance de taurillons N'Dama entretenus suivant divers modes d'embouche. Rev. Elev. Méd. vét. Pays trop., 1972, 25 (2) : $317-324$.

3. LHOSTE (P.). Embouche intensive des Zébus de l'Adamaoua. I. Comparaison de différents systèmes d'alimentation (1970). Rev. Elev. Méd. vét. Pays trop., 1972, 25 (2) : 259-280.

4. PIOT (J.). Rapports annuels 1967-68-69-70-71. Station fourragère de Wakwa, Cameroun.

5. PIOT (J.). Etudes pastorales en Adamaoua Camerounais. Rev. Elev. Méd. vét. Pays trop., 1966, 19 (1) : 45-62.

6. PIOT (J). Cultures fourragères et élevage extensif. Conditions de l'Adamaoua. Colloque Elevage OCAM, Fort-Lamy, déc. 1969. 